\title{
Systemic capillary leak syndrome following granulocyte colony-stimulating factor therapy in a T-lymphoblastic leukemia/lymphoma patient: a case report
}

\author{
Ahmed H. Al Sharie (iD - Yazan O. Al Zu’bi - Sarah Al Sharie (D) Hawra A. Baydoun · Farah H. Atawneh • \\ Osama Alshari (D) Dima Albals
}

Received: 9 September 2021 / Accepted: 3 December 2021 / Published online: 21 January 2022

(C) The Author(s), under exclusive licence to Springer-Verlag GmbH Austria, part of Springer Nature 2022

\begin{abstract}
Summary
Introduction Systemic capillary leak syndrome (SCLS) is a rare and often fatal clinical entity used to describe a generalized increase in vascular permeability leading to fluid extravasation toward the interstitial compartment. SCLS could be an idiopathic disease or secondary to infections, malignancies or drugs.

Case We present a case of presumably granulocyte colony-stimulating factor (G-CSF)-induced SCLS in a 21-year-old man diagnosed with T-lymphoblastic leukemia/lymphoma. He received the 6th cycle (part B) of the hyper-CVAD chemotherapeutic regimen followed by the initiation of neutropenic fever prophylaxis protocol which included antibiotics and G-CSF. In a course of hours, the patient became dyspneic, hypotensive, and edematous which required intensive care unit admission and was stabilized ac-
\end{abstract}

The authors Ahmed H. Al Sharie and Yazan O. Al Zu'bi contributed equally to the manuscript.

A. H. A. Sharie - Y. O. A. Zu'bi · H. A. Baydoun

Faculty of Medicine, Jordan University of Science and Technology, 22110 Irbid, Jordan

\section{S. A. Sharie}

Faculty of Medicine, Yarmouk University, 22110 Irbid, Jordan

\section{F. H. Atawneh}

Department of Medical Laboratory Sciences, Faculty of Applied Medical Sciences, Jordan University of Science and Technology, Irbid, Jordan

\section{O. Alshari $(\varangle)$}

Division of Oncology, Department of Internal Medicine, Faculty of Medicine, Jordan University of Science and Technology, P.O. Box: 3030, 22110 Irbid, Jordan osamasalshari@gmail.com

\section{Albals}

Department of Medicinal Chemistry and Pharmacognosy, Faculty of Pharmacy, Yarmouk University, Irbid, Jordan cordingly. In the following days the patient's anasarca progressively increased which was associated with hypoalbuminemia, hypotension and anemia with pericardial and bilateral plural effusions. As a diagnosis of exclusion augmented by the acuity of such clinical event, observed concomitantly with the administration of the prophylaxis protocol, the suspicion of G-CSF-induced SCLS was established. Consequently, G-CSF was discontinued and treatment with dexamethasone and intravenous immunoglobulins (IVIG) was started. The patient's condition improved significantly illustrated by hemodynamic stability in addition to improvement regarding the anasarca, hypoalbuminemia, and anemia. Follow-up scans suggest resolution of the pericardial and plural effusions.

Conclusion SCLS remains a serios and potentially fatal complication of G-CSF administration which should be taken into consideration, since such medication is widely utilized in oncology wards.

Keywords SCLS - Computed tomography · Granulocyte colony-stimulating factor · G-CSF . Intravenous immunoglobulins · IVIG

\section{Introduction}

SCLS is a rare and fatal angiopathy characterized by reversable protein leakage into the interstitial space leading to a classical triad of hypotension, hypoalbuminemia and hemoconcentration [1]. The exact incidence of SCLS is unknow since this disease entity is diagnostically challenging and usually misdiagnosed or mislabeled with a wide range of diagnoses including sepsis, idiopathic anaphylaxis, ovarian hyperstimulation syndrome, pancreatitis, protein-losing enteropathy, angioedema, Gleich syndrome, toxic shock syndrome, hemophagocytic lymphohistiocyto- 
sis, and many others [2, 3]. The pathophysiological mechanism leading to SCLS is poorly studied but hypothesized to be a state of endothelial dysfunction and hypercytokinemia [3]. A considerable number of case reports have elucidated that SCLS can be precipitated by certain medications including vincristine [4], gemcitabine [5], rituximab [6], trastuzumab [7], acitretin [8], clofarabine [9], interleukin-2 [10], interleukin-11 [11], and (G-CSF) [12]. The latter being the core of our article as we report a case of G-CSFinduced SCLS in a 21-year-old patient diagnosed with T-lymphoblastic leukemia/lymphoma. These reports are extremely important to elucidate the therapeutic plan used in a life-threating, rare and unexpected condition.

\section{Case presentation}

A 21-year-old Mediterranean man diagnosed with T-lymphoblastic leukemia/lymphoma was admitted to receive the 6th cycle in the hyper-CVAD (part B) protocol. Basic admission laboratory investigations revealed marked leukocytosis $\left(59.80 \times 10^{3} / \mathrm{mm}^{3}\right)$ with a differential of: neutrophils (79\%), myelocytes (4\%), metamyelocytes (9\%), bands (5\%), lymphocytes (1\%), monocytes (1\%), basophile (1\%) and eosinophils (0\%), normocytic anemia (hemoglobin: $9.60 \mathrm{~g} / \mathrm{dL}$, hematocrit: $28.60 \%$, mean cell volume: $86.20 \mu \mathrm{m}^{3}$ ), elevated lactate dehydrogenase levels $(896.0 \mathrm{U} / \mathrm{L})$ with normal kidney and liver function tests. The patient was doing well since the last chemotherapy cycle (5th cycle) with no fever spikes associated with good appetite and no weight loss. Systemic review of symptoms was unremarkable except for constipation which was treated accordingly. The patient developed severe neutropenia (white blood cell count: $0.30 \times 10^{3} / \mathrm{mm}^{3}$ ) in the 5 th cycle and was treated with G-CSF. The 6 th cycle regimen consisted of methotrexate $(200 \mathrm{mg}$, intravenously [IV]) over $2 \mathrm{~h}$ followed by $800 \mathrm{mg}$ over the next $24 \mathrm{~h}$ on the first day. Succeeded by cytarabine $(3 \mathrm{~g})$ given IV over $2 \mathrm{~h}$ every $12 \mathrm{~h}$ on the second and third days. Concurrently, methylprednisolone (50 mg, IV) was given from day one to three. On the second day, intrathecal methotrexate $(12 \mathrm{mg})$ was also injected simultaneously. Methotrexate levels were measured to be $0.13 \mu \mathrm{mol} / \mathrm{L}$ in which administration of folinic acid has started upon the end of methotrexate infusion with a dose of $15 \mathrm{mg}$, IV every $6 \mathrm{~h}$. Finally, the protocol was planned to end by the injection of intrathecal cytarabine $(100 \mathrm{mg})$ on the eighth day. The oncology team has decided to start the febrile neutropenia prophylaxis protocol on the seventh day as the patient developed previously multiple attacks of neutropenic fever triggered by the same chemotherapeutic agents. The patient did not have any signs or symptoms of infection. Febrile neutropenia was documented in cycles 1, 3 and 4 that was treated according to the current guidelines with G-CSF and antibiotics. The protocol was not changed since it induced an ex- cellent regression of his malignancy. The prophylactic protocol consisted of teicoplanin (400 mg, Q24, IV), meropenem (1 g, Q8, IV), and G-CSF $(5 \mu \mathrm{g} / \mathrm{kg} / \mathrm{h}, \mathrm{SC})$ given simultaneously. G-CSF was used after the onset of febrile neutropenia in previous cycles while in cycle 6 , it was used prophylactically.

On the same day, the patient became lethargic, dyspneic, hypotensive, edematous with an oxygen saturation of $88 \%$ on room air with normal temperature. Complete blood count illustrated a normocytic anemia (hemoglobin: $9.3 \mathrm{~g} / \mathrm{dL}$, hematocrit: $27.5 \%$, mean cell volume: $83.8 \mu^{3}$ ) with normal white blood cell count $\left(3.5 \times 10^{3} / \mathrm{mm}^{3}\right)$ and platelets count $\left(145 \times 10^{3} / \mathrm{mm}^{3}\right)$. Kidney function test included creatinine $(35.00 \mu \mathrm{mol} / \mathrm{L})$, urea $(5.30 \mathrm{mmol} / \mathrm{L})$, sodium $(136.0 \mathrm{mmol} / \mathrm{L})$ and potassium $(4.4 \mathrm{mmol} / \mathrm{L})$ levels. Liver function test included alanine aminotransferase $(23.1 \mathrm{U} / \mathrm{L})$, aspartate transaminase $(14 \mathrm{U} / \mathrm{L})$, alkaline phosphatase $(129 \mathrm{U} / \mathrm{L})$, gamma glutamyl transferase $(26 \mathrm{U} / \mathrm{L})$, albumin $(35 \mathrm{~g} / \mathrm{L})$, total protein $(57 \mathrm{~g} / \mathrm{L})$, total bilirubin $(12.2 \mu \mathrm{mol} / \mathrm{L})$ and direct bilirubin $(3.6 \mu \mathrm{mol} / \mathrm{L})$. The decision was made to transfer the patient to the intensive care unit and treated accordingly with IV fluids and vasopressors, which resulted in transient short-term improvements. Consequently, the intrathecal cytarabine dose was omitted and the febrile neutropenia prophylaxis protocol was continued. In the next $24 \mathrm{~h}$, the patient's condition continued to worsen as the patient was still hypotensive, tachycardiac, tachypneic and edematous, with marginal low oxygen saturation on oxygen therapy with an unknown etiology for the clinical picture. Laboratory investigations only illustrated normocytic anemia (hemoglobin: $8.9 \mathrm{~g} / \mathrm{dL}$, hematocrit: $26.20 \%$, mean cell volume: $83.40 \mu^{3}$ ) with negative septic workup including blood, urine and cerebrospinal fluid cultures. On the next day, the patient condition deteriorated with a blood pressure of $95 / 48 \mathrm{~mm}$ $\mathrm{Hg}$, heart rate of $131 \mathrm{bpm}$, and a respiratory rate of 20 BPM. Laboratory investigations showed a marked decrease in the hematocrit to $21.2 \%$ and hemoglobin of $7.4 \mathrm{~g} / \mathrm{dL}$, white blood cell count of $0.2 \times 10^{3} / \mathrm{mm}^{3}$, with an absolute neutrophil count of 48.8. A remarkable hypoalbuminemia $(29.4 \mathrm{~g} / \mathrm{L})$ with low total protein of $48.3 \mathrm{~g} / \mathrm{L}$ was also observed compared to 34.4 and $58.2 \mathrm{~g} / \mathrm{L}$, respectively, on the previous day. Kidney and liver function tests were all within the normal limits. Urine analysis was also normal with no proteinuria. Repeated blood cultures for aerobic and anaerobic microorganisms alongside urine culture were also negative. Coagulation profile showed values of $\mathrm{D}$-dimer $(3.38 \mu \mathrm{g} / \mathrm{mL})$, fibrin degradation product $(3.38 \mu \mathrm{g} / \mathrm{mL})$, fibrinogen $(797.00 \mathrm{mg} / \mathrm{dL}), \mathrm{PT}$ (15.3s), PTT (36.8 s) and INR (1.23).

Chest computed tomography (CT) scan with IV contrast showed severe pericardial effusion with a maximum thickness of $4.5 \mathrm{~cm}$ with enhancing pericardium associated with bilateral pleural effusion more on the left side and bilateral atelectatic changes (Fig. 1a, b). 

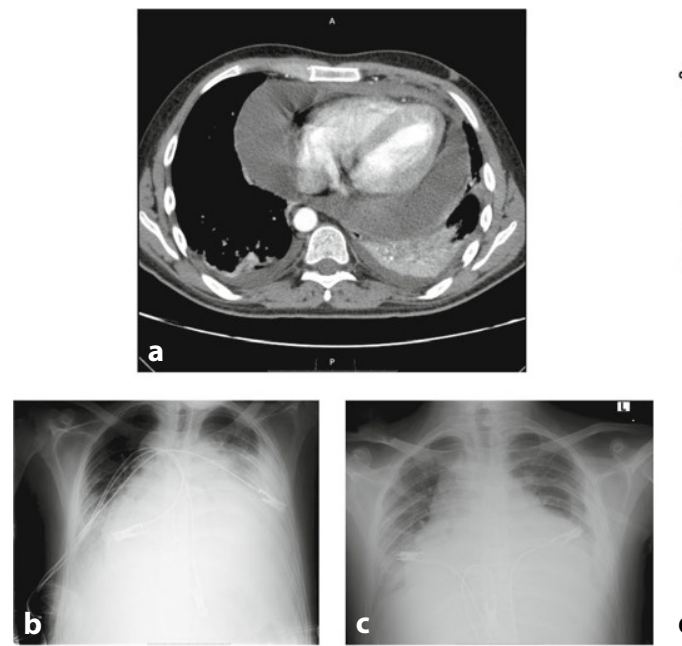

Fig. 1 Computed tomography (CT) scan with intravenous (IV) contrast showed severe pericardial effusion with a maximum thickness of $4.5 \mathrm{~cm}$ with enhancing pericardium associated with bilateral pleural effusion more on the left side and bilateral atelectatic changes on day 13 (a). Chest X-ray on day 14 (b). Chest X-ray on day 15 several hours after dexam-

In the next 2 days, supportive treatment was continued associated with progressive decline in albumin and hematocrit levels with no clinical improvement. The suspicion of G-CSF-induced SCLS was established as a diagnosis of exclusion, since G-CSF therapy had been used multiple times after previous chemotherapeutic cycles for neutropenic fever and severe neutropenia. The patient had developed mild edema and shortness of breath after each therapeutic session. These clinical findings were monitored and treated conservatively. We believe that these episodes were a result of milder form of G-CSF-induced SCLS that was amplified during the 6th cycle. G-CSF was immediately stopped and the patient was treated with dexamethasone (2 mg, Q6, IM) and IVIG $(400 \mathrm{mg} / \mathrm{kg}$, Q24, IV). Fig. 1c represents a chest X-ray following the initiation of the therapeutic plan. Darbepoetin alfa injection $(0.45 \mu \mathrm{g} / \mathrm{kg}$, SC, once weekly, as appropriate) was also started. The discrepancy between hypoalbuminemia and the lack of hemoconcentration is explained by the severe chemotherapy-induced bone marrow suppression as the patient required almost daily blood transfusions. Fig. $1 \mathrm{~d}$ represents the daily changes after admission in albumin, hematocrit, white blood cell (WBC) count and absolute neutrophil count. During the next 10 days, the patient exhibited good progression including stable vitals, increase in hematocrit and hemoglobin to $32.1 \%$ and $10.9 \mathrm{~g} / \mathrm{dL}$ respectively, increase in WBC count and absolute neutrophil count to $3.9 \times 10^{3}$ and 2882.1 , respectively. Albumin levels reached $38 \mathrm{~g} / \mathrm{L}$. Another chest CT scan with IV contrast showed significant improvement in the pericardial effusion with a maximum thickness of $1 \mathrm{~cm}$. Left-sided pleural effusion with subsegmental collapse consolidation in the lower left lung is also seen (Fig. 2a). Another scan was performed a month later suggesting complete resolution of the pleural and pericardial effusions without atelectatic changes observed (Fig. 2b).

\section{Discussion}

SCLS is an exceptionally rare and often fatal disease characterized by reversible plasma leakage into the interstitial space, and vascular collapse accompanied by hemoconcentration (elevated hemoglobin and hematocrit) and hypoalbuminemia [2]. In 1960, Dr. Bayard Clarkson described SCLS for the first time, and through 2016, there had been fewer than 500 cases documented in the literature $[13,14]$. In any case, it becomes clear that this condition is underreported due to the fact that it is commonly misdiagnosed owing to its resemblance in signs and symptoms with other prevalent conditions including sepsis, angioedema, and anaphylaxis, as well as its nonspecific signs and symptoms, not to mention the high mortality rates $[2,13]$.

Capillary leak syndrome acute episode consists of three phases: first it begins with the prodromal phase which is characterized by conventional nonspecific symptoms and deterioration of the general status, i.e., flu-like symptoms including cough, myalgia, and rhinorrhea, in addition to digestive symptoms including abdominal pain, vomiting and diarrhea [15]. Subsequently, a sudden and a conceivably fatal hypovolemia occurs during the leak phase which is portrayed by weight gain and generalized edema. The consequences are numerous and serious, ranging from hypovolemic shock and cardiac arrest to multiple organ dysfunction syndrome and systemic 

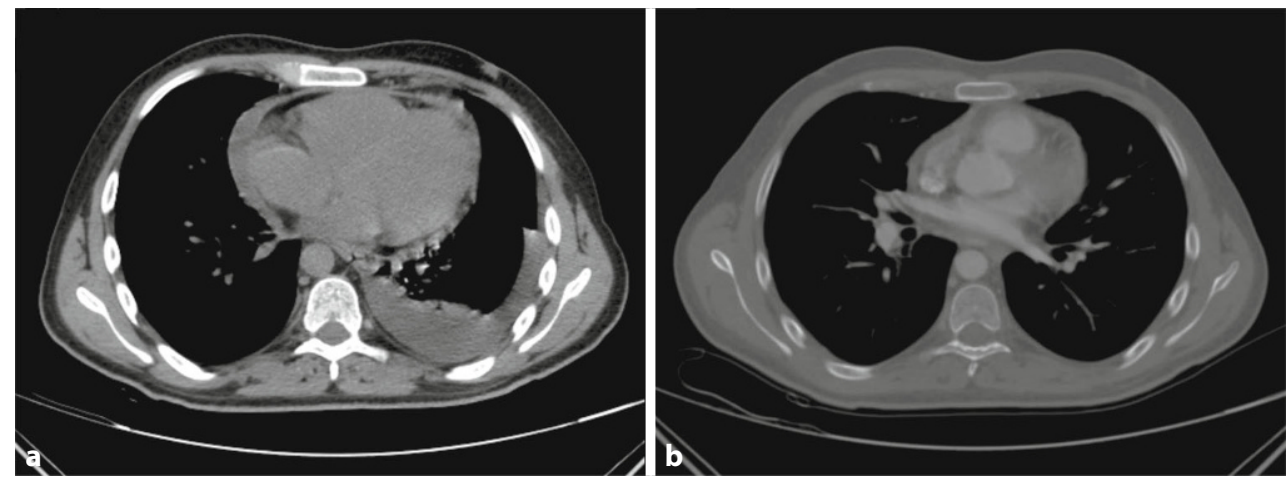

Fig. 2 Chest computed tomography (CT) scan on day 24 after admission showing significant improvement in the pericardial effusion with a maximum thickness of $1 \mathrm{~cm}$. Left-sided pleural effusion with subsegmental collapse consolidation in the lower left lung (a). Follow-up chest CT 1 month after the previous scan revealed complete resolution of pericardial and pleural effusions with no atelectatic changes (b) inflammatory response syndrome [15]. Ultimately, a post-leak phase with restoration of blood pressure and abolishment of edema with massive polyuria commences. However, a distinguishable pulmonary edema, which is often fatal, is elicited by the iatrogenic fluid overload used in the management [15]. The diagnosis of SCLS is purely clinical since there are no specific biomarkers or diagnostic tests [13].

The exact etiology of the disease remains unknown, yet abundant mechanisms have been proposed as being potential explanations to this phenomenon. In vitro, the serum of SCLS patients induced microvascular endothelial cell apoptosis via oxidation injury as a suggestive mechanism, with a remarkable increase in reactive oxygen species [16]. It has been shown that high concentrations of pro-adrenomedullin are present in the serum of SCLS patients during acute attacks of the disease, to which hypotension can be regarded [17]. Although there is acontemporaneous presence of monoclonal gammopathy of unknown significance (MGUS) in $70-85 \%$ of SCLS patients, its role in the flare ups remains unclear as it is seen in primary and secondary SCLS [18, 19]. Furthermore, angiopoietin-2 which induces vascular inflammation and activation, neo-angiogenesis, and leakage was found to be elevated in a case reported by Yamanouchi et al., which is consistent with previous findings in the literature where it was elevated in SCLS patients' sera, whereupon the inhibition of angiopoietin-2 impeded the permeability induced in vivo $[20,21]$. With regard to G-CSF, it is thought that the stimulated and increased population of granulocytes results in vascular endothelial damage due to extravagant production of superoxide anion free radicles resulting in leakage and increased permeability, since G-CSF stimulates the proliferation and differentiation of granulocytes in addition to enhancing the expression of adhesion molecules [22].

SCLS can be idiopathic which is generally known as Clarkson's disease. Yet, it can be secondary to multiple factors, including drugs, malignancies, infections, and autoimmune diseases, with $44 \%$ of cases having a recognizable cause behind the attacks $[13,15]$. Multiple drugs have been found to provoke SCLS, Mertz et al. reported the drugs associated using VigiBase, the World Health Organization Global Individual Case Safety Report (ICSR) database, in which they found a predomination of immunomodulating and antineoplastic agents, namely gemcitabine, clofarabine, and denileukin diftitox, to be related with the greatest number of cases [23]. G-CSF was the most frequently reported drug inducing SCLS in another report on 62 cases [24]. In addition, hematological malignancies have shown to have a greater risk of SCLS and its associated mortality when compared with non-hematological malignancies, with the most common being non-Hodgkin lymphoma and multiple myeloma [24]. Incidentally, SCLS can be the presenting manifestation of a malignancy, e.g., in the literature, cases of anaplastic large cell lymphoma, large B-cell lymphoma, and T-cell lymphoma have been reported as initially presenting with SCLS [25-27]. Viral infections explicitly have been linked to SCLS including Ebola, dengue, Hantaviruses, Marburg, influenza A and more recently SARS-CoV-2 (severe acute respiratory syndrome coronavirus 2) [15]. Concerning autoimmune and autoinflammatory diseases, psoriasis has been the most commonly reported, followed by systemic lupus erythematosus and antiphospholipid syndrome $[28,29]$.

Despite the scarcity, several reports of G-CSF-induced SCLS can be found in the literature - to the best of our knowledge only eight publications describing 12 cases [12, 30-36]. Nakagawa et al. uniquely described an instance of idiopathic SCLS with elevated G-CSF levels that were correlated with the clinical course of the disease treated with theophylline, $\beta-2$ agonists alongside prednisolone; as a result, G-CSF levels were reduced by the action of steroids which improved the patient's condition accordingly [37]. Two cases of peripheral blood stem cells donors with insignificant past medical history developed SCLS 
after G-CSF administration [34, 36]. Dagdemir et al. reported a case of a pediatric patient diagnosed with acute non-lymphoblastic leukemia who has been administered chemotherapy and G-CSF, and consequently developed the attack on the fourth day of G-CSF therapy, but she did not develop hemoconcentration during this attack [30]. Similarly, our patient had severe suppression resulting in normocytic anemia due to an inconsistency in our hematocrit (21.2\%) and hemoglobin $(7.4 \mathrm{~g} / \mathrm{dL})$ findings-which showed a decline rather than being elevated; for this reason he was given packed red blood cells and darbepoetin alfa. Despite the discrepancy, our case shares some similarities with previous reports including hypotension, negative cultures, hypoxia, pulmonary and pericardial effusions, edema and fever [30, 31, 33, 35].

Due to the fact that the etiology of SCLS remains unclear, the treatment is empiric with no prophylactic treatments available and the most commonly utilized treatments are methylxanthines, $\beta$-agonists and prednisone [38, 39]. Our patient was given intravenous fluids, dexamethasone, darbepoetin alfa and IVIG, to which the patient response was favorable as evidenced by the stable vitals, elevated albumin, hematocrit, and hemoglobin levels described previously. This lends support to previous findings in the literature in which IVIG has shown to be effective in two patients, in which IVIG reduced the patient's weight and hematocrit levels along with enhancing the serum albumin levels [40]. SCLS remains a fatal complication of G-CSF administration which should be taken into consideration during management, since such medication is highly used in oncology [41].

\section{Conclusion}

We report a case of G-CSF-induced SCLS seen in a 21-year-old man diagnosed with T-lymphoblastic leukemia/lymphoma. The main goal of the manuscript is to draw attention to a serious, rare and fatal complication of G-CSF therapy. In addition, SCLS laboratory findings in our case were atypical-lacking the hemoconcentration due to severe bone marrow suppression which was previously reported and explained in other cases. Furthermore, the utilization of dexamethasone and IVIG in the management of SCLS is emphasized.

Author Contribution Y.O.A, A.H.A, S.A, and H.A.B wrote the first draft of the manuscript. F.H.A, O.A, and D.A. were the main investigators involved in the patient's care, collecting and analyzing patients' data and critically reviewed the manuscript. All authors read, revised and approved the final version of the manuscript.

\section{Declarations}

Conflict of interest A.H. A. SharieX, Y.O. A. Zu'bi, S.A. Sharie, H.A. Baydoun, F.H. Atawneh, O. Alshari and D. Albals declare that they have no competing interests.
Ethical standards This report has been conducted and written in accordance to the ongoing regulations for case reports and case series in the King Abdullah University Hospital (KAUH). Case reports are exempted from institutional ethical approval by the institutional review board (IRB). Consent to publish the case report was not obtained because the manuscript does not contain any personal information that could lead to the identification of the patient according to HIPPA guidelines.

\section{References}

1. Rajan $\mathrm{R}$, et al. Idiopathic systemic capillary leak syndrome-an often missed diagnosis. Trop Doct. 2021;51(2):246-8.

2. Druey KM, Greipp PR. Narrative review: the systemic capillaryleaksyndrome. Ann Intern Med. 2010;153(2):90-8.

3. Siddall E, Khatri M, Radhakrishnan J. Capillary leak syndrome: etiologies, pathophysiology, and management. KidneyInt. 2017;92(1):37-46.

4. Zhang Y-T, et al. Unexpected vincristine-induced systemic capillary leak syndrome in patients with Wilm's tumor: a single institution experience. Pediatr Hematol Oncol. 2020;37(1):90-6.

5. Bajwa R, Starr J, Daily K. Gemcitabine-induced chronic systemic capillary leak syndrome. BMJ Case Rep. 2017;2017:bcr2017221068.

6. Vedala K, et al. Capillary leak syndrome from rituximab therapy oflymphoma. J Investig Med High Impact Case Rep. 2020;8:2324709620942372.

7. Zhang F, Yang J, Li Z. Trastuzumab-induced systemic capillary leak syndrome in a breast cancer patient. Pathol Oncol Res. 2014;20(2):435-7.

8. Vos LE, Vermeer MH, Pavel S. Acitretin induces capillary leak syndrome in a patient with pustular psoriasis. J Am AcadDermatol. 2007;56(2):339-42.

9. Baytan B, et al. Clofarabine-induced capillary leak syndrome in a child with refractory acute lymphoblastic leukemia. J Pediatr Hematol Oncol. 2010;32(2):144-6.

10. Dhupkar P, Gordon N. Interleukin-2: old and new approaches to enhance immune-therapeutic efficacy. In: Naing A, Hajjar J, editors. Immunotherapy. Cham: Springer; 2017. pp. 33-51.

11. Liu Z, Li D, Zhu Y. Interleukin-11-induced capillary leak syndrome companied with abdominal chylous leakage in primary sigmoid carcinoma patients with thrombocytopenia. JCan Res Ther. 2015;11(4):1024-1024.

12. Oeda E, et al. Capillary leak syndrome likely the result of granulocyte colony-stimulating factor after high-dose chemotherapy. Intern Med. 1994;33(2):115-9.

13. Druey KM, Parikh SM. Idiopathic systemic capillary leak syndrome (Clarkson disease). J Allergy Clin Immunol. 2017;140(3):663-70.

14. Clarkson B, et al. Cyclical edema and shock due to increased capillary permeability. Am J Med. 1960;29(2):193-216.

15. Bichon A, et al. Capillary leak syndrome: state of the art in 2021. Rev Med Interne. 2021;42(11):789-96. https://doi. org/10.1016/j.revmed.2021.05.012.

16. Assaly R, et al. Initial evidence of endothelial cell apoptosis as a mechanism of systemic capillary leak syndrome. Chest. 2001;120(4):1301-8.

17. Xie Z, et al. Adrenomedullin surges are linked to acute episodes of the systemic capillary leak syndrome (Clarkson disease). JLeukoc Biol. 2018;103(4):749-59.

18. Hakeem RM, Druey K, Zhihui X. The role of monoclonal paraproteins in systemic capillary leak syndrome (SCLS). J Immunol. 2016;196(1):124-58. 
19. Pineton de Chambrun $\mathrm{M}$, et al. Intravenous immunoglobulins improve survival in monoclonal gammopathy-associated systemic capillary-leak syndrome. Am J Med. 2017;130(10):1219.e19-1219.e27.

20. XieZ, et al. Vascular endothelial hyperpermeabilityinduces the clinical symptoms of Clarkson disease (the systemic capillary leak syndrome). Blood. 2012;119(18):4321-32.

21. Yamanouchi S, et al. Dysregulation of angiopoietin-1 and angiopoietin-2 in an infant with fatal Clarkson disease. Pediatr Int. 2020;62(12):1400-1.

22. Shinohara K. Systemic capillary leak syndrome caused by granulocyte colony-stimulating factor. Intern Med. 2011;50(19):2259-2259.

23. Mertz P, et al. Characterizing drug-induced capillary leak syndromes using the world health organization vigibase. JAllergy Clin Immunol. 2019;143(1):433-6.

24. Shin JI, et al. Systemic capillary leak syndrome (Clarkson syndrome) in cancer patients: a systematic review. J Clin Med. 2018;7(11):418.

25. Lourdes LS, et al. Systemic capillary leak syndrome as an initial presentation of ALK-negative anaplastic large cell lymphoma. Case Rep Hematol. 2012;2012:954201.

26. Umemoto T, et al. Capillaryleak syndrome: initial presentation in a patient with ALK+ anaplastic large cell lymphoma associated with increased levels of serum cytokines. Leuk Lymphoma. 2011;52(6):1139-42.

27. Pothen L, et al. Systemic capillary leak syndrome revealing a diffuse large B-cell lymphoma. Acta Clin Belg. 2014;69(4):305-8.

28. Takimoto Y, et al. Gamma/delta T cell lymphoma presenting in the subcutaneous tissue and small intestine in a patient with capillary leak syndrome. Int J Hematol. 1998;68(2):183-91.

29. Bressan AL, et al. Systemic capillary leak syndrome. An Bras Dermatol. 2011;86(3):593-5.

30. Dagdemir A, et al. G-CSF related capillary leak syndrome in a child with leukemia. Leuk Lymphoma. 2001;42(6):1445-7.

31. Rechner I, Brito-Babapulle F, Fielden J. Systemic capillary leak syndrome after granulocyte colony-stimulating factor (G-CSF). Hematol J.2003;4(1):54-6.

32. Rechner I, Brito-Babapulle F, Fielden J. Systemic capillary leak syndrome after granulocyte colony-stimulating factor (G-CSF). Hematol J. 2003;4(1):54-6.

33. Heitger A, et al. Capillary leak syndrome in a patient with septicemia and granulocyte-colony-stimulating factor $(\mathrm{G}$ -
CSF)-induced accelerated granulopoiesis. Med Pediatr Oncol. 1998;31(2):126-7.

34. de Azevedo AM, Goldberg Tabak D. Life-threatening capillary leak syndrome after G-CSF mobilization and collection of peripheral blood progenitor cells for allogeneic transplantation. Bone Marrow Transplant. 2001;28(3):311-2.

35. Deeren DH, Zachee P, Malbrain MLNG. Granulocyte colony-stimulatingfactor-inducedcapillaryleaksyndrome confirmed by extravascularlung water measurements. Ann Hematol. 2005;84(2):89-94.

36. Andersen P, Gertsen SA, Dickmeiss K. Capillary leak syndromein a healthy donor following GCSF stimulation. Bone MarrowTransplant. 2007;39:S140.

37. Nakagawa N, et al. A case of idiopathic systemic capillary leak syndrome with high serum levels of G-CSF on exacerbation. Intern Med. 2011;50(6):597-600.

38. Kapoor P, et al. Idiopathic systemic capillary leak syndrome (Clarkson's disease): the mayo clinic experience. Mayo Clin Proc. 2010;85(10):905-12.

39. Amoura Z, et al. Systemic capillary leak syndrome: report on 13 patients with special focus on course and treatment. Am J Med. 1997;103(6):514-9.

40. Lambert M, etal. High-dose intravenous immunoglobulins dramatically reverse systemic capillary leak syndrome. Crit Care Med. 2008;36(7):2184-7. https://doi.org/10.1097/ CCM.0b013e31817d7c71.

41. Alshari O, et al. Evaluating the prognostic role of monocytopenia in chemotherapy-induced febrile neutropenia patients treated with granulocyte colony-stimulating factor. Ther Clin RiskManag. 2021;17:963-73.

Publisher's Note Springer Nature remains neutral with regard to jurisdictional claims in published maps and institutional affiliations.

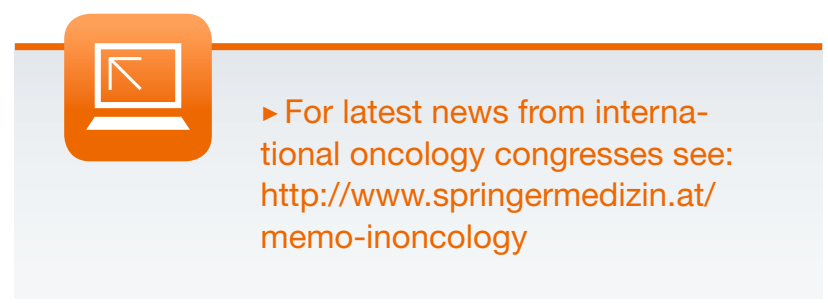

